\title{
Expectancy Induces Dynamic Modulation of Corticospinal Excitability
}

\author{
Gijs van Elswijk, Bert U. Kleine, Sebastiaan Overeem, \\ and Dick F. Stegeman
}

\begin{abstract}
Behavioral studies using motor preparation paradigms have revealed that increased expectancy of a response signal shortens reaction times (RTs). Neurophysiological data suggest that in such paradigms, not only RT but also neuronal activity in the motor structures involved is modulated by expectancy of behaviorally relevant events. Here, we directly tested whether expectancy of a response signal modulates excitability of the corticospinal system used in the subsequent movement. We combined single- and paired-pulse transcranial magnetic stimulation (TMS) over the primary motor cortex with a simple RT task with variable preparatory delays. We found that, in line with typical behavioral observations, the subjects' RTs
\end{abstract}

\section{INTRODUCTION}

Studies in both human and animal subjects have shown that cognitive factors influence neuronal information processing at several levels in the motor system, including the primary motor cortex (Georgopoulos, 2000) and the spinal cord (Bizzi, Tresch, Saltiel, \& d'Avella, 2000). Preparation paradigms have been used extensively to study cognitive motor processes separately from the actual motor execution (for reviews, see Riehle, 2005; Requin, Brener, \& Ring, 1991). A well-studied preparation paradigm is the simple reaction time (RT) task. In that task, two successive signals are presented to the subject: a preparatory signal (PS), followed by a response signal (RS) that gives an instruction to move. The interval between the two signals is called the preparatory delay. Many behavioral studies have shown convincingly that increased expectancy of an RS raises the subject's readinessto-respond, as is reflected in shortened RTs (see, for example, Requin et al., 1991; Luce, 1986; Niemi \& Näätänen, 1981; Näätänen, 1972; Gordon, 1967). Simple RT tasks with variable preparatory delays are very effective to experimentally manipulate RS expectancy (and therefore, readiness-to-respond), namely, via the conditional probability of the RS. The conditional probability of an

Radboud University Nijmegen Medical Centre, Nijmegen, The Netherlands decreased with increasing response signal expectancy. TMS results revealed a modulation of corticospinal excitability in correspondence with response signal expectancy. Besides an increased excitability over the time-course of the preparatory delay, corticospinal excitability transiently increased whenever a response signal was expected. Paired-pulse TMS showed that this modulation is unlikely to be mediated by excitability changes in interneuronal inhibitory or facilitatory networks in the primary motor cortex. Changes in corticospinal synchronization or other mechanisms involving spinal circuits are candidates mediating the modulation of corticospinal excitability by expectancy.

event is the probability that this event will occur at a certain point in time, given that it has not occurred yet.

Several neurophysiological studies have used simple RT tasks with variable preparatory delays to investigate effects of RS expectancy in the motor system. From research on nonhuman primates, we have learned that both firing rate and synchronization between cortical neurons are strongly influenced by the probability that an RS will occur (Janssen \& Shadlen, 2005; Riehle, Grün, Diesmann, \& Aertsen, 1997). Furthermore, it has recently been demonstrated in humans that the strength of oscillatory synchronization between the motor cortex and spinal cord neurons, in the gamma frequency range ( 40 to $70 \mathrm{~Hz}$ ), is closely correlated with RS expectancy (Schoffelen, Oostenveld, \& Fries, 2005). Increasing RS probability also increases the amplitude of the contingent negative variation, a cortical potential associated with motor preparation (Trillenberg, Verleger, Wascher, Wauschkuhn, \& Wessel, 2000). Negative cortical potentials have been claimed to result from excitatory postsynaptic potentials at the apical dendrites of pyramidal neurons (Brunia, 1993; Birbaumer, Elbert, Canavan, \& Rockstroh, 1990), which would imply that RS expectancy increases excitability of cortical motor areas. Together, these data, obtained with diverse neurophysiological techniques, strongly suggest that expectancy of an RS modulates excitability of motor structures that are used in an upcoming movement. We therefore hypothesize 
that during movement preparation, excitability of the prime mover cortical representation adapts dynamically to the probability of an expected RS.

In humans, the main technique to probe directly and noninvasively excitability of the corticospinal system is single-pulse transcranial magnetic stimulation (TMS). The ability of single-pulse TMS to measure modulation of corticospinal excitability has been put to use in a large number of RT studies. For example, shortly after an RS is given, corticospinal excitability rapidly increases, starting about 100 msec before the voluntary muscle response (Yamanaka et al., 2002; Leocani, Cohen, Wassermann, Ikoma, \& Hallett, 2000; Chen, Yaseen, Cohen, \& Hallett, 1998; Rossini, Zarola, Stalberg, \& Caramia, 1988).

Paired-pulse TMS is often used as a complementary technique to single-pulse TMS. In paired-pulse TMS, a subthreshold conditioning pulse is followed by a suprathreshold test pulse. Depending on the interval between the conditioning pulse and the test pulse, the efficacy of the test pulse is either suppressed or facilitated. Typically, the muscle response to the test pulse is inhibited if intervals of $1-5$ msec are used, whereas intervals of 10-15 msec are facilitatory (Chen, Tam, et al., 1998; Ziemann, Rothwell, \& Ridding, 1996; Kujirai et al., 1993). Furthermore, as this modulation takes place at a cortical rather than at a spinal level (Ziemann et al., 1996; Kujirai et al., 1993), the paired-pulse technique can be used to further specify the mechanisms underlying excitability changes measured with single-pulse TMS.

Here, we investigated whether corticospinal excitability is modulated by expectancy of an RS. Therefore, we combined the variable delay paradigm (Trillenberg et al., 2000; Riehle et al., 1997; Requin et al., 1991) with direct measurements of corticospinal excitability by means of singleand paired-pulse TMS over the primary motor cortex.

\section{METHODS}

\section{Experiment 1}

In the first experiment, we used single-pulse TMS to investigate the effects of RS expectancy on the dynamics of corticospinal excitability during a variable delay simple reaction task.

\section{Subjects}

Nine healthy volunteers (8 women and 1 man), aged $19-29$ years $($ mean $=22.7)$, participated in the experiment. All were right-handed, with a mean handedness score of $94(S D=9)$, according to the Oldfield questionnaire (Oldfield, 1971), and had normal or correctedto-normal vision. Subjects were screened for any history of neurological illness or neurosurgery, and for any metal or electronic implants. All subjects gave written informed consent prior to the experiment. All experimental procedures were approved by the local ethics committee and were in accordance with the Declaration of Helsinki.

\section{Procedure and Task}

The experimental task was a simple abduction of the index finger. Four preparatory delays and catch trials were presented in random order with equal a priori probability, but with an increasing conditional probability. As a consequence, there were four moments within each trial, indicated on the computer screen, where the subject could expect an instruction to move. Hence, the subjects were able to accurately anticipate the increasingly probable RS, but they were not certain whether an RS would occur after one of the delays or not at all.

The experiment was controlled by stimulus software written in MATLAB (The MathWorks, Inc., Natick, MA) using the Psychophysics Toolbox (Brainard, 1997), running on a Pentium-III class PC. The subject sat in a chair in front of a 15-inch computer screen (distance $\sim 75 \mathrm{~cm}$ ). A custom-built isometric force meter (Zijdewind \& Kernell, 1994) was placed on the armrests of the chair. Both hands lay in pronated position with the elbow flexed to about 90 degrees. The right-hand index finger was placed inside the force meter. At the beginning of the session, the force meter was calibrated to the subject's maximum voluntary force at isometric abduction of the right index finger. Force was digitized (1000 samples/sec) using an isometric strain gauge force transducer and fed back to the stimulus software.

During the task, on-line feedback of time and force was provided via a cursor on the computer screen. A cartoon of a fish was used as the force/time cursor (for details, see Figure 1 and its legend). At the beginning of each trial, the subject was required to completely relax his or her hand muscles. When force had been at resting level for two consecutive seconds, the cursor started moving. The onset of horizontal cursor movement indicated the beginning of preparatory delay and will from here be referred to as the preparatory signal (PS). PS-RS delays of $1000,1500,2000$, or $2500 \mathrm{msec}$ were randomly intermixed, with probabilities of 1/5 each, corresponding to a total of 36 trials per RS delay. In the remaining $1 / 5$ of trials, the PS was not followed by an RS (catch trials). Time-courses of all trial types used in the experiment are depicted schematically in Figure 2A. If the subject made an error, on-line feedback was provided at the end of the trial to encourage optimal performance. Possible errors were false alarms, unlikely fast responses $(<80 \mathrm{msec})$, too slow responses $(>600 \mathrm{msec}$ ), failure to maintain force at resting level until RS onset, or failure to reach the required force level during the response.

In $2 / 3$ of the trials, a single TMS pulse was applied within the preparatory delay. Trials with or without TMS were presented in random order. The magnetic stimulus was given: (a) just before one of the moments were an RS could occur (980, 1480, 1980, or 2480 msec after the 


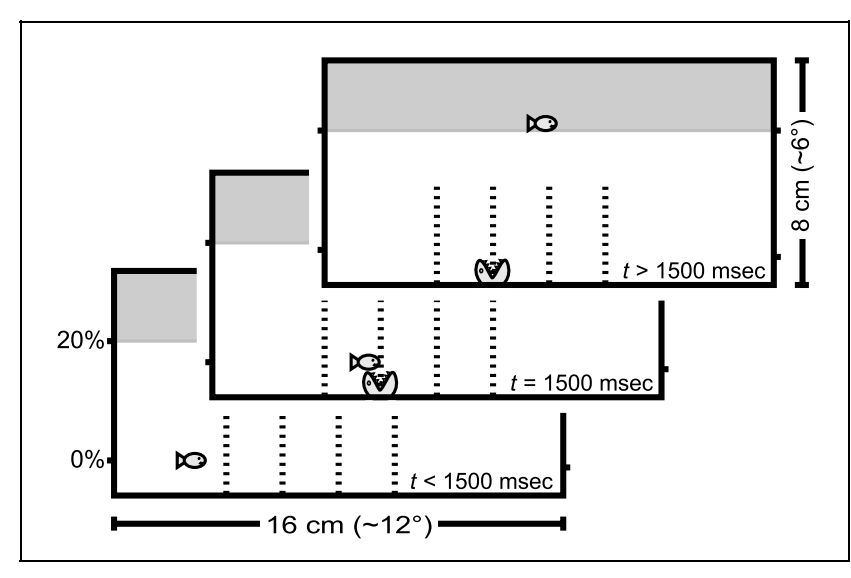

Figure 1. Schematic of the stimulus display during one of the trials of the experiment. The vertical position of the fish depended on the force applied to the force meter, with $4.2 \mathrm{~cm}$ (visual angle of $\sim 3.2$ degrees) corresponding to $20 \%$ of the subject's maximum force. The horizontal position was determined by the time-point within the trial: The fish moved horizontally with a velocity of $4.4 \mathrm{~cm} / \mathrm{sec}(\sim 3$ degrees $/ \mathrm{sec})$. In addition to feedback of force and time via the cursor, required force levels and possible delay deadlines were marked. Vertical markers indicated the four possible delays. Approach of the fish to a marker thus corresponded to the approach of a possible deadline. A cartoon of a monster was used as the RS. The monster could appear only at the position of one of the vertical markers, just below the current position of the fish. The subject was instructed to avoid the monster and to move the fish, as quickly as possible, into the upper area of the screen, which had a distinct background color. In order to move the fish into that area, subjects needed to abduct their index finger to at least $20 \%$ of their maximum force.

PS), (b) just before the midpoint between two moments where an RS could occur (1230, 1730, or 2230 msec after the PS), (c) well before any RS could occur (730 msec after the PS). Stimulation times were chosen pseudorandomly such that TMS always preceded the RS. Over the entire experiment, 15 TMS pulses were given at each stimulation time. Subjects were asked to ignore the possible interference of TMS on task performance as much as they could.
The experiment consisted of six blocks of 30 trials. The first two trials of each block were considered warm-up trials and were excluded from the analysis. Between two successive blocks, there were a few minutes of rest. During a rest break the mean RTs of all completed blocks were shown on the computer screen and the investigator encouraged subjects to improve their RT in upcoming blocks. In addition, the subject was notified by the investigator if too much muscle activity was observed in the trial phases that required muscle relaxation.

Prior to the experimental session, all subjects completed a training session. The training session was equivalent to the experimental session, except that in the training session no TMS was applied and that auditory feedback of the electromyogram (EMG) was provided to the subjects. The EMG feedback was given to assist in practicing muscle relaxation. In addition, at the end of the training session, subjects who were naive to TMS were familiarized with the technique. The training session and the experimental session took about $2 \mathrm{hr}$ each and were conducted on separate days.

\section{Electromyography}

EMG was recorded using $10-\mathrm{mm}$ diameter $\mathrm{Ag}-\mathrm{AgCl}$ selfadhesive surface electrodes (Kendall-LTP, Chicopee, MA). Electrodes were placed on the first dorsal interosseus (FDI) of the right hand in a "belly-tendon" arrangement, following standard skin preparation. The signal was continuously monitored, amplified (ActiveOne amplifier; BioSemi, Amsterdam, The Netherlands), bandpass filtered $(10-500 \mathrm{~Hz})$, digitized (2048 samples/sec), and stored for off-line analysis using MyoDAQ software (Blok, van Dijk, Drost, Zwarts, \& Stegeman, 2002).

\section{Transcranial Magnetic Stimulation}

Subjects wore a tightly fitting Lycra swimming cap. To attenuate the clicking sound associated with the

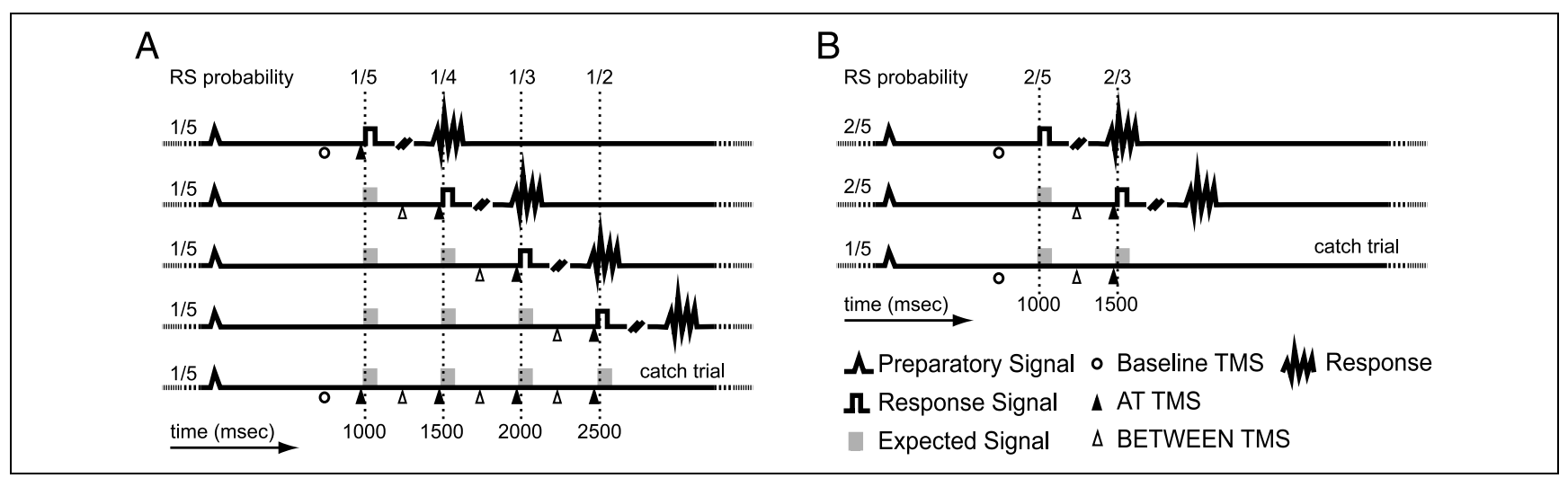

Figure 2. Schematic representation of the time-courses of the trial types used in Experiment 1 (A) and Experiment 2 (B). In Experiment 1 , TMS was applied in 2/3 trials. In Experiment 2, TMS was applied in 116/200 trials. A priori RS probabilities are listed vertically, whereas conditional RS probabilities are listed horizontally. 
magnetic stimulation, the subjects wore earplugs. TMS was delivered using a figure-of-eight shaped coil (diameter $70 \mathrm{~mm}$ ) connected to a Magstim 200 stimulator (Magstim, Whitland, UK). The coil was held tangentially on the left hemiscalp with its handle pointing backwards at an angle of about 45 degrees from the midsagittal axis.

At the beginning of the experimental session, the motor hotspot and the resting motor threshold of the right-hand FDI were determined for each subject. Surface markings were drawn onto the swimming cap to serve as reference points against which the coil was positioned. The motor hotspot was defined as the position at which the amplitude of motor-evoked potentials (MEPs) was the largest for a given stimulus intensity. After the motor hotspot was determined, TMS was applied at decreasing intensities to determine the subject's resting motor threshold (Rossini et al., 1994). The resting motor threshold was defined as the lowest stimulus intensity needed to produce an MEP with an amplitude greater than $50 \mu \mathrm{V}$ peak-to-peak, in at least five out of eight successive stimulations. The mean $(S D)$ motor threshold was 39\% (7\%) of the maximum stimulator output. During the experiment, the coil was held at the motor hotspot, and stimulation intensity was set to $110 \%$ of the individual motor threshold.

\section{Data Processing and Analysis}

Data were processed off-line. Trials in which subjects made any of the previously described errors (e.g., false alarms) were discarded. To check whether the target muscle was indeed at rest at the time of TMS, a 200-msec prestimulus EMG trace was inspected for each TMS trial. First, a computer program marked the trials in which the rectified trace contained any peak with amplitude of more than $25 \mu \mathrm{V}$. In addition, traces were visually inspected and subsequently marked if any prestimulus EMG activity was detected. All marked trials were discarded, effectively eliminating trials with any pre-TMS activity of the prime mover from the analyses. Furthermore, the root mean square (RMS) amplitude of the 200-msec pre-TMS EMG trace was calculated for each TMS trial.

RT was defined as the duration between the presentation of the RS and the onset of voluntary EMG. We defined two RT conditions: (1) the trials without TMS were assigned to the NO-TMS condition; (2) the trials with TMS were assigned to the TMS condition. RTs were averaged over replications, for each subject, condition, and delay. RTs below $100 \mathrm{msec}$ were excluded from the analysis.

MEP amplitude was defined as the difference between the lowest and highest values of the raw EMG signal (peak-to-peak amplitude) within the time window of $20-40$ msec after the TMS trigger. In order to reduce the between-subjects variability, a standardization procedure was employed. The mean and standard devia- tions of the MEPs observed at the earliest stimulation time (730 msec after the PS) for each subject were used as a baseline for that subject. This baseline was chosen because it was located outside the range of the RS delays used, and thus, at this point no RS ever occurred. The individual amplitudes of the MEPs observed for each subject at the other stimulation times were converted into $z$-scores calculated from the baseline (cf. Hasbroucq, Kaneko, Akamatsu, \& Possamai, 1999). TMS could occur in two distinct situations: almost at the same time as a possible RS, or at time points not associated with an RS. We therefore defined two MEP conditions: (1) The "AT" condition for trials where TMS was applied at 980, 1480, 1980, or $2480 \mathrm{msec}$ after the PS, and (2) The "BETWEEN" condition for trials where TMS was applied at 1230, 1730, or $2230 \mathrm{msec}$ after the PS (see Figure 2A). The $z$-transformed MEP amplitudes and raw pre-TMS RMS amplitudes were averaged over replications for each subject, condition, and stimulation time.

RTs, pre-TMS RMS amplitudes, and MEP amplitudes were analyzed separately. The analyses were based on a repeated-measures multiple regression (Lorch \& Myers, 1990). In each semipartial correlation ( $s r$ ) reported, all remaining predictors have been partialed out from the predictor under analysis. For statistical tests, the mean square of the interaction between the subject and the factor under analysis was used as the error term. Statistical significance was set at the .05 level. Unless stated otherwise, data are presented as mean \pm standard deviation.

\section{Experiment 2}

In the second experiment, we investigated whether the facilitatory effect of RS expectancy on MEP amplitude, as apparent from Experiment 1, can be attributed to changes in intracortical excitability. Therefore, singleand paired-pulse TMS were applied in a paradigm similar to Experiment 1. Intracortical inhibition (ICI) and intracortical facilitation (ICF) were assessed using pairedpulse TMS with interpulse intervals of 2 and $10 \mathrm{msec}$, respectively. To make the experiment feasible, given the fact that three types of TMS were to be applied, only two RS delays were used.

\section{Subjects}

Seven healthy volunteers ( 5 women and 2 men), aged 22-30 years $($ mean $=26.6$ ), participated in the experiment. All were right-handed, with a mean handedness score of $96(S D=7)$, according to the Oldfield questionnaire (Oldfield, 1971), and had normal or correctedto-normal vision. Subjects were screened for any history of neurological illness or neurosurgery, and for any metal or electronic implants. All subjects gave written informed consent prior to the experiment. 


\section{Procedure and Task}

The experimental procedures and task were similar to Experiment 1, only the aspects that differ from the previous experiment are described here.

Two PS-RS delays of 1000 and 1500 msec were randomly intermixed with probabilities of $2 / 5$ each, corresponding to a total of 80 trials per RS delay. The remaining $1 / 5$ of the trials were catch trials (see Figure 2B).

In 116 out of the 200 trials, a TMS stimulus was applied within the preparatory delay. The magnetic stimulus was given: (a) just before the last RS could occur (1480 msec after the PS), (b) just before the midpoint between the two moments were an RS could occur (1230 msec after the PS), or (c) before any RS could occur (730 msec after the PS). Single and paired magnetic stimuli were presented pseudorandomly, using remote operation of the magnetic stimulator by the stimulus software. At time (a) and time (b), all three TMS types were applied equally often: single-pulse TMS, paired-pulse TMS with an interval of 2 and 10 msec were each applied 16 times at both stimulation times. At time (c) only single pulses were applied, 20 times in total.

The experiment consisted of five blocks of 42 trials each; the training consisted of four blocks of 42 trials each. The training session was completed on the same day as the experimental session, with a break of about $1 \mathrm{hr}$ between the end of the training and the start of the experimental session. Altogether, the training and the experiment took about $3 \mathrm{hr}$.

\section{Electromyography}

EMG recording procedures were identical to Experiment 1 .

\section{Transcranial Magnetic Stimulation}

TMS was similar to Experiment 1. The coil, however, was connected to a Magstim BiStim ${ }^{2}$ setup (Magstim, Whitland, UK), to allow for both single- and paired-pulse TMS. Furthermore, after determining the motor hotspot and resting motor threshold, three additional parameters were determined: (1) the stimulus intensity that yielded a test MEP amplitude of about $1 \mathrm{mV}$, on average; (2) the ICI conditioning stimulus intensity needed to inhibit the test MEP to an amplitude of about $0.5 \mathrm{mV}$, on average; (3) the ICF condition stimulus intensity needed to facilitate the test MEP to an amplitude of about $1.5 \mathrm{mV}$, on average. Given the dynamic nature of the task, we did not attempt to match single-pulse MEP amplitudes across conditions, as there is evidence that the test MEP amplitude has no effect on the amount of ICI (for a detailed discussion on this issue, see Coxon, Stinear, \& Byblow, 2006).
The mean $(S D)$ motor threshold, test pulse intensity, ICI conditioning pulse intensity, and ICF conditioning pulse intensity were 51\% (6\%), 60\% (8\%), 38\% (6\%), and $40 \%$ (4\%) of the maximum stimulator output, respectively.

\section{Data Processing and Analysis}

Data processing was identical to Experiment 1.

For the RT analysis, RTs not preceded by TMS were assigned to the NO-TMS condition; the trials with TMS applied $270 \mathrm{msec}$ before the RS (730 and $1230 \mathrm{msec}$ after the PS, for the two RS delays, respectively) were assigned to the TMS condition. Because there were no TMS pulses applied at $980 \mathrm{msec}$ after the PS, RTs with TMS applied at $20 \mathrm{msec}$ before the RS did not exist for the early RS delays. Therefore, the trials in which TMS was applied at $1480 \mathrm{msec}$ after the PS (i.e., $20 \mathrm{msec}$ before the second RS delay) were not included in the analysis. For each subject and condition, RTs were averaged over replications and submitted to a repeatedmeasures analysis of variance (ANOVA), with RS delay (1000 msec vs. $1500 \mathrm{msec}$ ) and stimulation (NO-TMS vs. TMS) as within-subject factors.

For the MEP analysis, the MEPs obtained at a stimulation time of $1230 \mathrm{msec}$ after the PS were assigned to the BETWEEN condition; those obtained at a stimulation time of $1480 \mathrm{msec}$ after the PS were assigned to the AT condition. For each subject and condition, $z$-transformed MEP amplitudes and raw pre-TMS RMS amplitudes were averaged over replications and submitted to a repeated-measures ANOVA with stimulation time (BETWEEN vs. AT) and TMS type (single-pulse vs. paired-pulse $2 \mathrm{msec}$ vs. paired-pulse $10 \mathrm{msec}$ ) as withinsubject factors. Furthermore, two paired $t$ tests were conducted to compare the single-pulse MEPs in the BETWEEN and the AT conditions with baseline.

Statistical significance was set at the .05 level.

\section{RESULTS}

\section{Experiment 1}

In total, $11 \%$ of all trials were discarded because subjects made errors (3\%), or pre-TMS EMG activity was detected (8\%).

\section{Reaction Times}

Representative EMG traces from one subject, showing a voluntary EMG response preceded by an MEP, are plotted in Figure 3. The overall mean RT was $209 \pm 32 \mathrm{msec}$. A linear regression model, including delay (1000, 1500, 2000, 2500), condition (NO-TMS, TMS), and Delay $\times$ Condition as within-subject factors, was fitted to the averaged RT data, $R^{2}=.79, F(12,59)=18.08, p<.001$. 
Figure 3. Representative EMG traces from two trials of one subject in Experiment 1. In both trials, the RS was presented after a delay of $1500 \mathrm{msec}$ (dashed vertical line). Shortly after the RS, the voluntary EMG burst starts. In both examples, TMS was applied. The stimulation times are depicted by the short vertical lines. In the upper trace, the stimulation time was $1230 \mathrm{msec}$ (BETWEEN condition); in the lower trace, it was 1480 msec (AT condition).

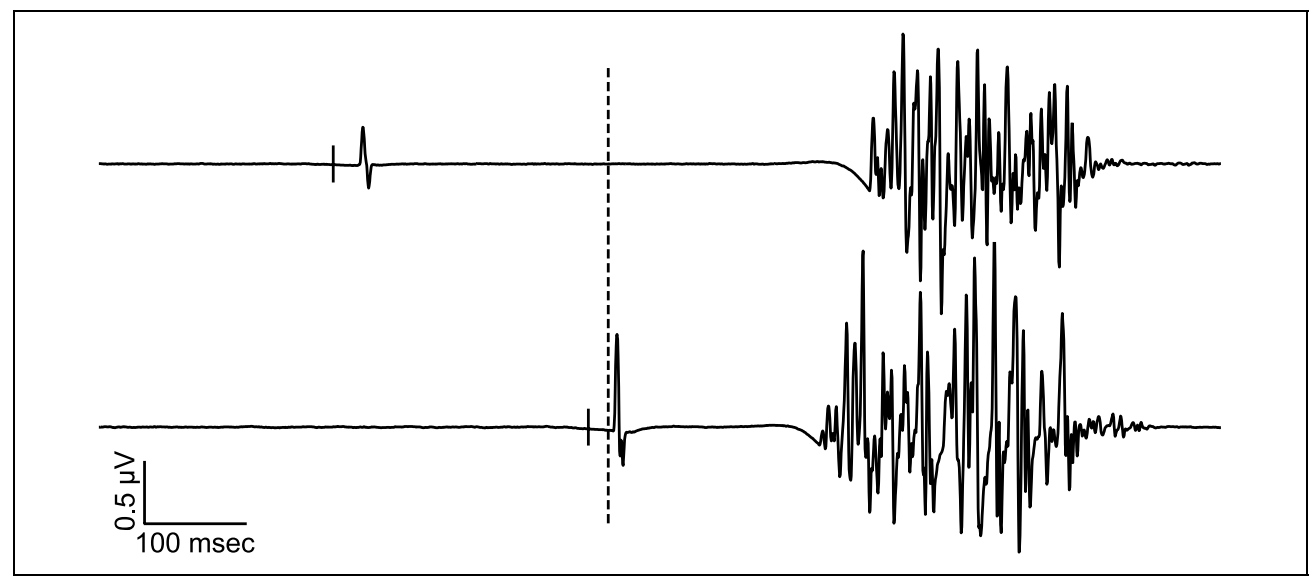

Figure 4 shows the RTs and the regression lines from the model. Analysis of the regression results showed that RT decreased significantly with increasing RS delay, $s r=-.51, F(1,8)=22.58, p<.01$. The RTs in the TMS condition were significantly shorter than in the NO-TMS condition, means, $199 \pm 31$ and $220 \pm 29 \mathrm{msec}$, respectively, $F(1,8)=25.78, p<$. 001 . There was no significant interaction between the effects of delay and condition, $F(1,8)=0.28, p>.1$.

For further insight in the facilitatory effect of TMS on RTs, an additional comparison was conducted between the RTs, separated into the AT and the BETWEEN TMS trials (data not shown in Figure 4). A paired $t$ test showed that there was no significant difference between the mean RTs in the AT and BETWEEN conditions, $197 \pm 38 \mathrm{msec}$ versus $202 \pm 29 \mathrm{msec}$, respectively; $t(35)=1.17, p>.1$.

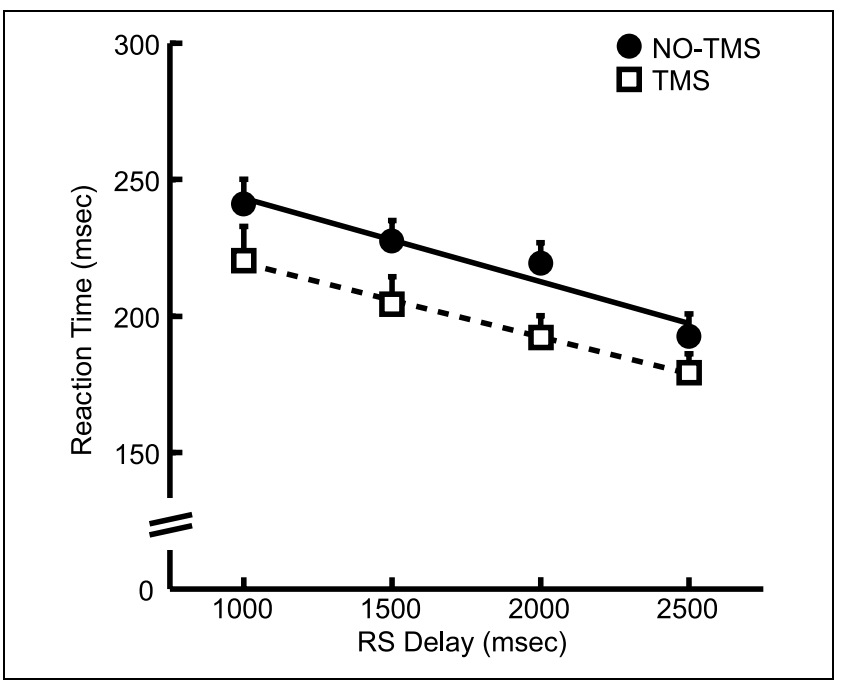

Figure 4. RT data of Experiment $1(n=9)$, with regression lines, plotted as a function of RS delay and condition. The markers represent the mean RT ( \pm standard error): filled circles for the reactions that were not preceded by a TMS pulse (NO-TMS condition) and open squares for the reactions that were preceded by a TMS pulse (TMS condition).

\section{Motor-evoked Potentials}

The overall mean raw MEP amplitude was $0.58 \pm 0.31 \mathrm{mV}$, the raw mean baseline MEP amplitude was $0.65 \pm$ $0.14 \mathrm{mV}$. A linear regression model, including stimulation time (980, 1230, 1480, 1730, 1980, 2230, $2480 \mathrm{msec}$ ), condition (AT, BETWEEN), and Stimulation time $\times$ Condition as within-subject factors, was fitted to the $z$-transformed MEP amplitudes, $R^{2}=.69, F(12,50)=9.36, p<.001$.

Figure 5 shows the average MEP amplitudes in both conditions as a function of stimulation time. The regression analysis revealed that MEP amplitudes increased significantly with increasing stimulation time, $s r=.28$, $F(1,8)=8.58, p<.05$. Furthermore, the amplitudes of MEPs obtained in the AT condition were significantly

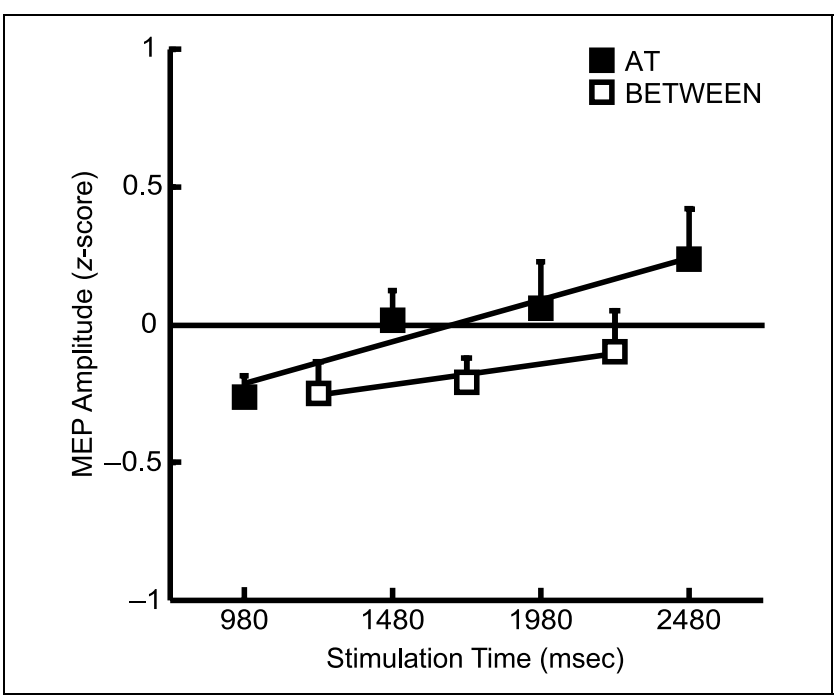

Figure 5. MEP data of Experiment $1(n=9)$, with regression lines, plotted as a function of stimulation time and condition. The markers represent the mean standardized MEP amplitudes ( \pm standard error): filled squares for the condition where TMS was applied AT expected RSs and open squares for the condition where TMS was applied BETWEEN expected RSs. At 1000, 1500, 2000, and 2500 msec (AT condition), an RS could occur; in between those moments (BETWEEN condition), RSs never occurred. 


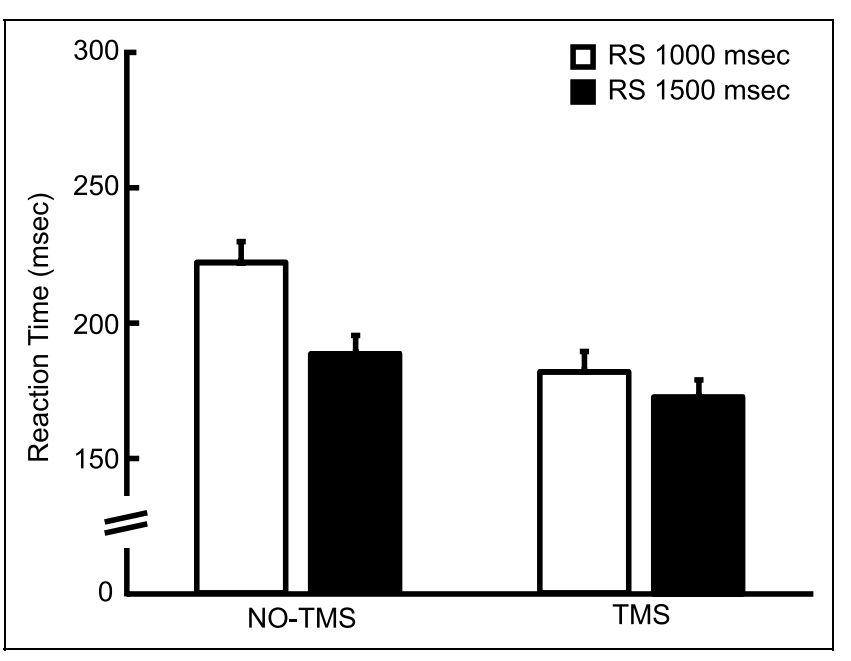

Figure 6. RT data of Experiment $2(n=7)$ plotted as a function of RS delay and condition. The bars represent the mean RT ( \pm standard error): open bars for the RS delays of $1000 \mathrm{msec}$, filled bars for the RS delays of 1500 msec.

larger than the amplitudes of MEPs in the BETWEEN condition, $F(1,8)=18.10, p<.01$. There was no significant Stimulation time $\times$ Condition interaction, $F(1,8)=$ 4.7, $p=.06$. The $y$-intercept of the regression line at $980 \mathrm{msec}$ significantly deviated from zero, indicating that the initial MEP amplitudes were below baseline, $F(1,8)=5.76, p<.05$.

The mean pre-TMS RMS amplitudes for stimulation times 980, 1230, 1480, 1730, 1980, 2230, and $2480 \mathrm{msec}$ were $2.7 \pm 0.9,2.6 \pm 0.9,2.7 \pm 1.0,2.8 \pm 1.0,2.7 \pm 1.0$, $2.9 \pm 1.0$, and $2.7 \pm 0.9 \mu \mathrm{V}$, respectively. A linear regression model, including the same predictors as used in the MEP regression, was fitted to these amplitudes, $R^{2}=.91, F(12,50)=43.69, p<.001$. The analysis of the regression results revealed no significant effects of stimulation time, condition, or Stimulation time $x$
Condition, $F(1,8)=1.41, p>.1 ; F(1,8)=0.36, p>.1$; $F(1,8)=2.65, p>.1$, respectively. Therefore, differences in corticospinal excitability cannot be explained by differences in EMG amplitude prior to the TMS pulse.

\section{Experiment 2}

In total, $14 \%$ of all trials were discarded because subjects made errors (5\%), pre-TMS EMG activity was detected $(7 \%)$, or there were technical problems (2\%).

\section{Reaction Times}

The overall mean RT was $191 \pm 14$ msec. Note that this is faster than in Experiment 1, likely due to the fact that the probabilities of the RSs were also higher. Figure 6 shows the RTs as a function of TMS condition and delay. The ANOVA showed a main effect of RS delay, indicating that in trials with an RS delay of $1500 \mathrm{msec}$, the responses were significantly faster than in trials with an RS delay of 1000 msec, means $181 \pm 16$ and $202 \pm$ 16 , respectively, $F(1,6)=12.66, p<.05$. In addition, the main effect of stimulation showed that the RTs in the TMS condition were significantly shorter than in the NO-TMS condition, means $177 \pm 18$ and $205 \pm$ 12 , respectively, $F(1,6)=39.52, p<.01$. No significant interaction between RS delay and TMS condition was found, $F(1,6)=4.19, p=.09$.

\section{Motor-evoked Potentials}

Representative EMG traces with MEPs from one subject are shown in Figure 7. The overall mean raw MEP amplitudes in the BETWEEN and AT conditions were $0.93 \pm 0.32 \mathrm{mV}$ in response to single-pulse TMS, $0.58 \pm$ $0.30 \mathrm{mV}$ in response to paired-pulse TMS with an ISI of $2 \mathrm{msec}$, and $1.33 \pm 0.40 \mathrm{mV}$ in response to paired-pulse
Figure 7. Representative EMG traces with MEPs of one subject in Experiment 2. One trace is shown for each of the six TMS conditions. Stimulation times are marked by short vertical lines. Note that in the ICI and ICF conditions, two TMS pulses are applied (a conditioning pulse followed by a test pulse). Within the single-pulse, ICI, and ICF conditions, the MEP amplitudes are larger in the AT trials than in the BETWEEN trials, indicating an increased corticospinal excitability. The

inhibitory effect of a conditioning pulse applied 2 msec prior to the test pulse can be seen by comparing the MEPs of the single-pulse and

ICI traces, whereas the facilitatory effect of a conditioning pulse applied $10 \mathrm{msec}$ prior to the test pulse can be seen by comparing the MEPs of the single-pulse and ICF traces.

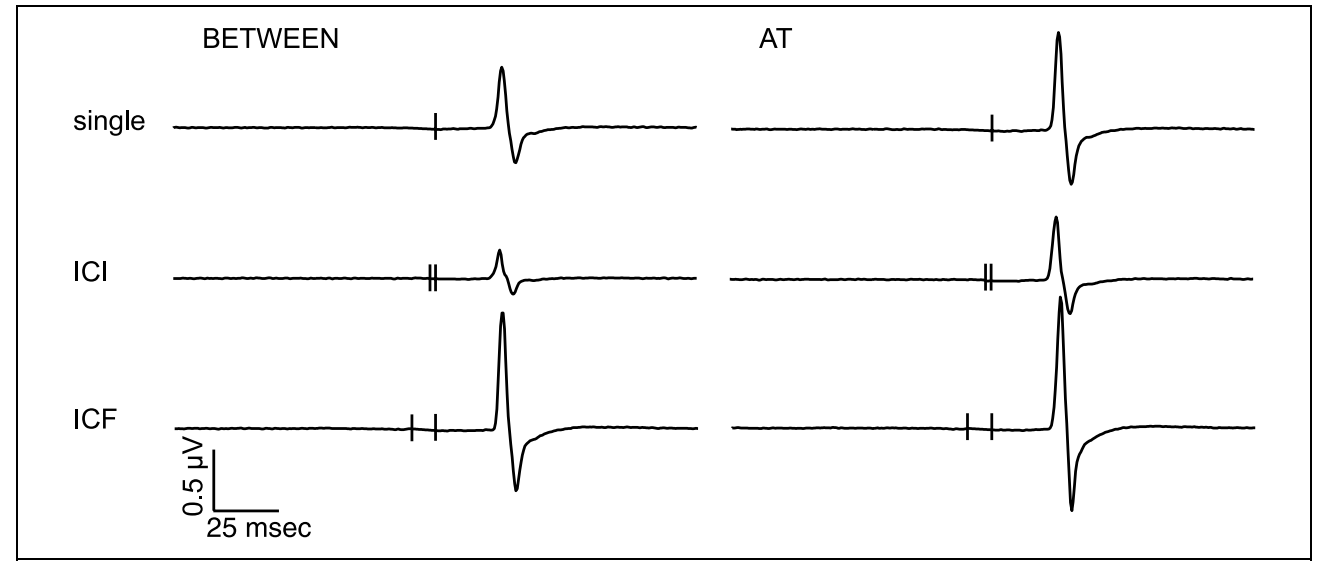


TMS with an ISI of 10 msec. The mean raw baseline MEP amplitude was $1.13 \pm 0.44 \mathrm{mV}$.

In Figure 8, the average $z$-transformed MEP amplitudes are plotted as a function of TMS type and stimulation time. The ANOVA revealed a significant main effect of TMS type on MEP amplitude, $F(2,12)=9.86$, $p<.01$. Planned contrasts confirmed that the MEP amplitudes were significantly smaller in response to pairedpulse TMS with an ISI of $2 \mathrm{msec}$, and were significantly larger in response to paired-pulse TMS with an ISI of $10 \mathrm{msec}$, compared to MEP amplitudes in response to single-pulse TMS, $F(1,6)=5.39, p<.05$ and $F(1,6)=$ $6.11, p<.05$, respectively. Furthermore, the main effect of stimulation time was significant, $F(1,6)=7.31, p<.05$, indicating that MEP amplitudes were larger when TMS was applied AT a moment where an RS was expected than when TMS was applied BETWEEN two expected RSs. Importantly, there was no significant interaction between TMS type and stimulation time, $F(2,12)=0.41$, $p>.1$, indicating that the effect of the conditioning pulse in both paired-pulse conditions (ICI and ICF) was not affected by RS expectancy. Similar to Experiment 1, single-pulse MEPs were significantly lower than baseline in the BETWEEN condition, $t(6)=-3.42, p<.05$, but not in the AT condition, $t(6)=0.10, p>.1$.

As an alternative approach to test whether RS expectancy differentially modulated ICI or ICF, we expressed the difference between paired-pulse MEP amplitudes and the average single-pulse MEP amplitude in each condition, as a percentage of that average single-pulse MEP amplitude. Paired $t$ tests again did not reveal an

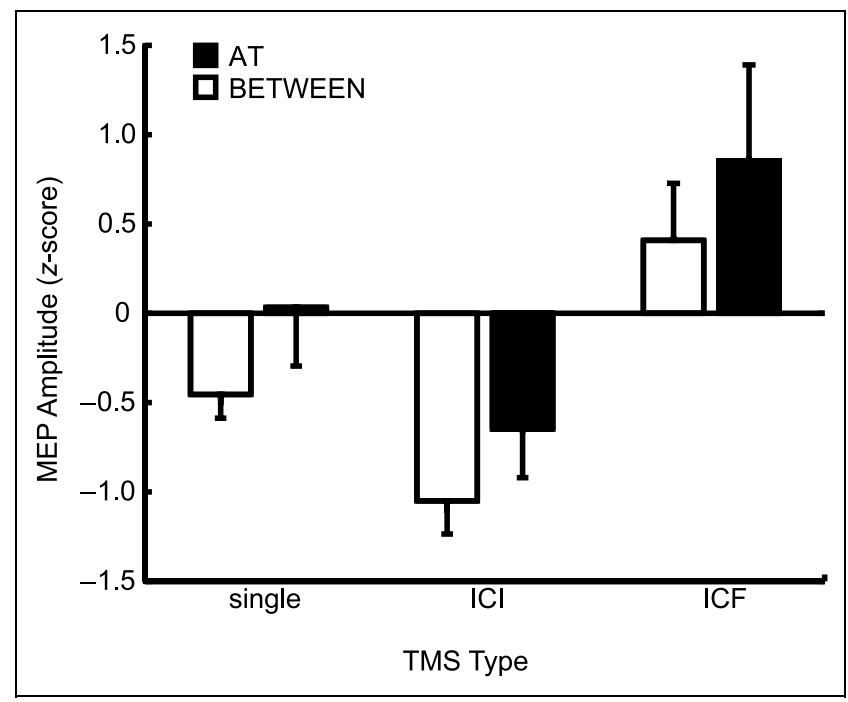

Figure 8. MEP data of Experiment $2(n=7)$ plotted as a function of stimulation time and TMS type. The bars represent mean standardized MEP amplitude ( \pm standard error): open bars for the condition where TMS was applied BETWEEN the expected RSs (stimulation time $1230 \mathrm{msec}$ ) and closed bars for the condition where TMS was applied AT an expected RS (stimulation time $1480 \mathrm{msec})$. effect of RS expectancy [means $\pm S E$, BETWEEN vs. AT for ICI: $-39.1 \pm 9.1 \%$ vs. $-29.5 \pm 10.7 \%, t(7)=-1.33$, $p=0.23$; for ICF: $67.0 \pm 21.3 \%$ vs. $45.8 \pm 25.5 \%, t(7)=$ $0.76, p=0.48]$.

The mean pre-TMS RMS amplitude in the singlepulse TMS trials was $3.0 \pm 0.8 \mu \mathrm{V}$ for the AT condition and $3.5 \pm 1.3 \mu \mathrm{V}$ for the BETWEEN condition. In the ICI trials, the pre-TMS RMS amplitude was $3.0 \pm 0.8 \mu \mathrm{V}$ for the AT condition and $3.3 \pm 1.3 \mu \mathrm{V}$ for the BETWEEN condition; in the ICF trials, it was $3.2 \pm 1.0 \mu \mathrm{V}$ for the AT condition and $3.1 \pm 0.9 \mu \mathrm{V}$ for the BETWEEN condition. The ANOVA on the pre-TMS RMS amplitudes revealed no significant effects of TMS type, stimulation time, or TMS type $\times$ Stimulation time, $F(2,12)=0.60, p>.1$; $F(1,6)=2.44, p>.1 ; F(2,12)=1.92, p>.1$, respectively. Therefore, differences in corticospinal excitability cannot be explained by differences in EMG amplitude prior to the TMS pulse.

\section{DISCUSSION}

Motor preparation facilitates efficient execution of goalbased behavior: The anticipation of relevant events in the environment allows us to react quickly once these actually occur. Here, we show that during movement preparation, excitability of the motor system is modulated by the mere expectancy of a behaviorally relevant event. More specifically, implicit knowledge about the probability that an instruction to move will occur shortens RT and elevates corticospinal excitability.

The analysis of MEP amplitudes revealed a modulation of corticospinal excitability that points to a complex interplay of several mechanisms (Figure 5). Foremost, MEP amplitudes were larger at time-points where an RS was expected compared with time-points where an RS could not occur. In addition, there was a nonspecific increase in MEP amplitudes over the time-course of the preparatory delay. Finally, modulation of MEPs initiated below baseline, suggesting the involvement of inhibitory mechanisms. We will first discuss the mechanisms that may account for the observed increases in MEP amplitudes.

Earlier neurophysiological work already showed that expectancy modulates neuronal activity in the motor system, suggesting that corticomotor excitability is modulated by the probability of behaviorally relevant events (Schoffelen et al., 2005; Thoenissen, Zilles, \& Toni, 2002; Trillenberg et al., 2000; Riehle et al., 1997). Our study is the first to use TMS to directly demonstrate that expectancy indeed increases corticospinal excitability and that this increase is transient, reflecting the capacity of the motor system to dynamically adapt the prime mover's excitability to current behavioral demands, on a timescale of tens of milliseconds.

There are several candidate mechanisms within the corticomotor system that may mediate the increase of 
corticospinal excitability, such as inhibitory or facilitatory interneuronal mechanisms within the primary motor cortex. For example, expectancy-induced increases in MEP amplitude could result from decreased excitability of intracortical inhibitory networks, increased excitability of intracortical facilitatory networks, or both. Pairedpulse TMS can probe such inhibitory (ICI) as well as facilitatory (ICF) networks, depending on the interval between the conditioning and the test pulse (Chen, Tam, et al., 1998; Ziemann et al., 1996; Kujirai et al., 1993). In Experiment 2, we used paired-pulse TMS with interpulse intervals of 2 and $10 \mathrm{msec}$ to assess ICI and ICF, respectively. In this experiment, a clear effect of RS expectancy on MEP amplitude was found again (Figure 8). However, there were no differential effects of RS expectancy across the three types of TMS stimuli applied within the task, making it unlikely that the facilitatory effect of RS expectancy on MEP amplitude is mediated by the intracortical networks tested here. It remains possible that modulation of ICI by RS expectancy would have been found if an interpulse interval other than 2 msec (e.g., $2.5 \mathrm{msec}$ ) had been applied because there is evidence that different intervals may probe different inhibitory mechanisms (Roshan, Paradiso, \& Chen, 2003; Fisher, Nakamura, Bestmann, Rothwell, \& Bostock, 2002). It must be noted, however, that Sohn et al. (2002) found that suppression of voluntary movements modulates ICI as measured with a 2 -msec interpulse interval. Thus, we have probed the same mechanism as in the study of Sohn and colleagues.

What mechanisms other than ICI or ICF may account for the observed changes in corticospinal excitability? Early studies have shown that spinal circuits are modulated during motor preparation (see also Brunia, 1993; Scheirs \& Brunia, 1985; Brunia, Scheirs, \& Haagh, 1982). It is unlikely that expectancy of response signals originates within the spinal cord, but expectancy may influence spinal excitability indirectly via projections originating from cortical areas. In a more recent study, it was shown that while monkeys were waiting for an RS, two concurrent processes took place in the interneurons of the spinal cord: excitatory rate-changes in the same direction as the subsequent movement related rate-changes, and inhibitory rate-changes that were hypothesized to reflect a superimposed mechanism suppressing muscular output (Prut \& Fetz, 1999). Accordingly, it has been put forward that the modulation of spinal circuits plays an important role in complex motor functions, such as the planning of movements (Bizzi et al., 2000). Future studies using more direct tests of spinal excitability (e.g., F-waves or H-reflexes) may shed light on the role of spinal mechanisms in our paradigm.

Corticospinal synchrony could be another mechanism underlying modulation of MEP amplitudes by RS expectancy. Our findings are in line with previous hypotheses that whenever a task-relevant event is expected, neurons that have to be activated in the anticipated task are tran- siently facilitated (Brunia, 1993; Birbaumer et al., 1990). If such anticipatory facilitation is subtle, it will not exceed the target neurons' firing thresholds, and therefore, not directly affect firing rates. Infraliminal facilitation may, however, lead to synchronization within the target neuron pools. Indeed, it has been shown that RS expectancy leads to increased synchronization within the primary motor cortex (Riehle et al., 1997), as well as between the motor cortex and the spinal cord (Schoffelen et al., 2005). Furthermore, neuronal synchronization has been proposed to determine the effectiveness of corticospinal interaction (Fries, 2005; Schoffelen et al., 2005). Thus, the MEP amplitude modulation observed in our experiments may be due to expectancy-induced changes in the interaction between neuronal groups of the primary motor cortex and the spinal cord: RS expectancy modulated neuronal synchronization, thereby shaping the susceptibility to TMS of target corticospinal pathways. Moreover, synchronization between distant neuronal groups in the primary motor cortex and the spinal cord may very well lead to increased MEP responses to suprathreshold TMS, but is unlikely to alter the effectiveness of the subthreshold conditioning pulse in paired-pulse TMS, which is in accordance with our findings (Figure 8).

In addition to the transient MEP facilitation by instantaneous RS expectancy, we found a weak but significant correlation between stimulation time and MEP amplitude in Experiment 1. Unexpectedly, this effect did not differ between the AT and the BETWEEN condition. If corticospinal excitability would solely be determined by the instantaneous expectancy of an RS, the MEP amplitudes at moments where no RS can occur (the BETWEEN condition) are not expected to modulate. This suggests the presence of an additional process (independent of and superimposed on the transient facilitation by each potential RS) reflecting the estimation of elapsed time, or the increase in the probability of encountering an RS, associated with the elapse of time in this paradigm (cf. Janssen \& Shadlen, 2005).

So far, we have discussed mechanisms that may account for the observed increases in MEP amplitudes. Our results also point to the involvement of an inhibitory mechanism. A few earlier TMS studies, which used constant preparatory delays, have reported inhibition of MEPs during motor preparation (Hasbroucq, Kaneko, et al., 1999; Hasbroucq, Osman, et al., 1999; Touge, Taylor, \& Rothwell, 1998; Hasbroucq, Kaneko, Akamatsu, \& Possamai, 1997). In our experiments we found similar effects: the modulation of the MEPs in Experiment 1 initiated below baseline; in Experiment 2, the MEP amplitudes in the BETWEEN condition were also suppressed relative to baseline. This implies an inhibitory mechanism that is employed shortly before the first RS is expected. Although our study cannot provide conclusive data on this assumption, we would like to speculate that this inhibition is closely related to proposed response suppression mechanisms (Coxon et al., 2006; 
Prut \& Fetz, 1999; Touge et al., 1998; Brunia, 1993; Birbaumer et al., 1990). Several studies have shown that ICI contributes to the voluntary suppression of a prepared movement (Coxon et al., 2006; Sohn, Wiltz, \& Hallett, 2002) and that ICI declines shortly before or during the execution of a voluntary movement (Buccolieri, Abbruzzese, \& Rothwell, 2004; Reynolds \& Ashby, 1999). Together with the results of Experiment 2, this suggests that ICI does not mediate the anticipatory facilitation of corticospinal excitability but rather serves as a countermechanism to such potential influences until the instruction to move is given. In the present experiment, it is very likely that inhibitory mechanisms were needed to prevent premature muscle output. Subjects were instructed and trained to pay much attention to muscle relaxation, while at the same time encouraged to respond as quickly as possible. Because at each potential RS the excitability of the corticospinal system was transiently facilitated, the subjects had to employ sufficient inhibition to make sure that these fluctuations in excitability remained subthreshold and did not produce premature muscle output.

A prominent feature of TMS is its ability to affect RTs, for example, due to intersensory facilitation or direct excitation of the motor pathways (Terao et al., 1997; Pascual-Leone et al., 1992). One can argue that the RTs in our experiment are influenced by such effects. To control for any bias of TMS on RTs, in both experiments, no TMS was applied in at least one third of the trials, and these trials were randomly intermixed with the TMS trials. Subjects had no prior information about the condition a given trial belonged to. The data from the conditions without TMS provide a reliable confirmation that our paradigm successfully modulated readiness-torespond: RTs systematically decreased with increasing RS delay (Figures 4 and 6). Thus, the subjects were capable of adjusting their readiness-to-respond in accordance with the probability that an RS could occur. It must be noted, however, that although TMS shortened the RTs, the facilitatory effect of RS delay on the RTs remained unchanged. Moreover, this RT speed-up was not different between the AT and BETWEEN trials of Experiment 1, suggesting that it resulted from intersensory facilitation (Terao et al., 1997).

To conclude, we postulate that readiness-to-respond is tuned by a well-controlled balance between levels of corticospinal facilitation and inhibition. Expectancy of response-related events dynamically increases excitability of the prime mover corticospinal representation, facilitating fast responses. Inhibitory mechanisms may counterbalance these excitatory effects in order to prevent premature output. An interesting issue that needs to be pursued is whether expectancy-related changes in neuronal synchronization in the motor system (Schoffelen et al., 2005; Riehle et al., 1997) hold any causal relation with expectancy-related changes in excitability as measured with TMS. Future experiments com- bining measures of neuronal synchronization with a direct measure of excitability, such as TMS, will provide valuable insights in this intriguing question.

\section{Acknowledgments}

This research was supported by grant 051.02 .050 of the Netherlands Organisation for Scientific Research (NWO). We thank Femke Maij for assistance with data collection; Hans van Dijk and Mark Massa for technical support; and Marieke van der Linden and Ton Dijkstra for helpful comments on earlier versions of the manuscript.

Reprint requests should be sent to Gijs van Elswijk, Department of Clinical Neurophysiology, Radboud University Nijmegen Medical Centre, PO Box 9101, NL-6500 HB Nijmegen, The Netherlands, or via e-mail: gijs@vanelswijk.net.

\section{REFERENCES}

Birbaumer, N., Elbert, T., Canavan, A. G. M., \& Rockstroh, B. (1990). Slow potentials of the cerebral cortex and behavior. Physiological Reviews, 70, 1-41.

Bizzi, E., Tresch, M. C., Saltiel, P., \& d'Avella, A. (2000). New perspectives on spinal motor systems. Nature Reviews Neuroscience, 1, 101-108.

Blok, J. H., van Dijk, J. P., Drost, G., Zwarts, M. J., \& Stegeman, D. F. (2002). A high-density multichannel surface electromyography system for the characterization of single motor units. Review of Scientific Instruments, 73, 1887-1897.

Brainard, D. H. (1997). The psychophysics toolbox. Spatial Vision, 10, 433-436.

Brunia, C. H. M. (1993). Waiting in readiness: Gating in attention and motor preparation. Psychophysiology, 30, 327-339.

Brunia, C. H. M., Scheirs, J. G., \& Haagh, S. A. (1982). Changes of Achilles tendon reflex amplitudes during a fixed foreperiod of four seconds. Psychophysiology, 19, 63-70.

Buccolieri, A., Abbruzzese, G., \& Rothwell, J. C. (2004). Relaxation from a voluntary contraction is preceded by increased excitability of motor cortical inhibitory circuits. Journal of Physiology, 558, 685-695.

Chen, R., Tam, A., Butefisch, C., Corwell, B., Ziemann, U., Rothwell, J. C., et al. (1998). Intracortical inhibition and facilitation in different representations of the human motor cortex. Journal of Neurophysiology, 80, 2870-2881.

Chen, R., Yaseen, Z., Cohen, L. G., \& Hallett, M. (1998). Time course of corticospinal excitability in reaction time and self-paced movements. Annals of Neurology, 44, 317-325.

Coxon, J. P., Stinear, C. M., \& Byblow, W. D. (2006). Intracortical inhibition during volitional inhibition of prepared action. Journal of Neurophysiology, 95, 3371-3383.

Fisher, R. J., Nakamura, Y., Bestmann, S., Rothwell, J. C., \& Bostock, H. (2002). Two phases of intracortical inhibition revealed by transcranial magnetic threshold tracking. Experimental Brain Research, 143, 240-248.

Fries, P. (2005). A mechanism for cognitive dynamics: Neuronal communication through neuronal coherence. Trends in Cognitive Sciences, 9, 474-480. 
Georgopoulos, A. P. (2000). Neural aspects of cognitive motor control. Current Opinion in Neurobiology, 10, $238-241$.

Gordon, I. E. (1967). Stimulus probability and simple reaction time. Nature, 215, 895-896.

Hasbroucq, T., Kaneko, H., Akamatsu, M., \& Possamai, C. A. (1997). Preparatory inhibition of cortico-spinal excitability: A transcranial magnetic stimulation study in man. Cognitive Brain Research, 5, 185-192.

Hasbroucq, T., Kaneko, H., Akamatsu, N., \& Possamai, C. A. (1999). The time-course of preparatory spinal and cortico-spinal inhibition: An H-reflex and transcranial magnetic stimulation study in man. Experimental Brain Research, 124, 33-41.

Hasbroucq, T., Osman, A., Possamai, C. A., Burle, B., Carron, S., Depy, D., et al. (1999). Cortico-spinal inhibition reflects time but not event preparation: Neural mechanisms of preparation dissociated by transcranial magnetic stimulation. Acta Psychologica, 101, 243-266.

Janssen, P., \& Shadlen, M. N. (2005). A representation of the hazard rate of elapsed time in macaque area LIP. Nature Neuroscience, 8, 234-241.

Kujirai, T., Caramia, M. D., Rothwell, J. C., Day, B. L., Thompson, P. D., Ferbert, A., et al. (1993). Corticocortical inhibition in human motor cortex. Journal of Physiology, 471, 501-519.

Leocani, L., Cohen, L. G., Wassermann, E. M., Ikoma, K., \& Hallett, M. (2000). Human corticospinal excitability evaluated with transcranial magnetic stimulation during different reaction time paradigms. Brain, 123, 1161-1173.

Lorch, R. F., \& Myers, J. L. (1990). Regression analyses of repeated measures data in cognitive research. Journal of Experimental Psychology: Learning, Memory, and Cognition, 16, 149-157.

Luce, R. D. (1986). Response times: Their role in inferring elementary mental organization. New York: Oxford University Press.

Näätänen, R. (1972). Time uncertainty and occurrence uncertainty of the stimulus in a simple reaction time task. Acta Psychologica, 36, 492-503.

Niemi, P., \& Naätanen, R. (1981). Foreperiod and simple reaction-time. Psychological Bulletin, 89, 133-162.

Oldfield, R. C. (1971). Assessment and analysis of handedness: Edinburgh inventory. Neuropsychologia, 9, 97-113.

Pascual-Leone, A., Valls-Sole, J., Wassermann, E. M., Brasil-Neto, J., Cohen, L. G., \& Hallett, M. (1992). Effects of focal transcranial magnetic stimulation on simple reaction time to acoustic, visual and somatosensory stimuli. Brain, 115, 1045-1059.

Prut, Y., \& Fetz, E. E. (1999). Primate spinal interneurons show pre-movement instructed delay activity. Nature, 401, 590-594.

Requin, J., Brener, J., \& Ring, C. (1991). Preparation for action. In J. R. Jennings \& M. G. H. Coles (Eds.), Handbook of cognitive psychophysiology (pp. 357-448). Chichester: Wiley.

Reynolds, C., \& Ashby, P. (1999). Inhibition in the human motor cortex is reduced just before a voluntary contraction. Neurology, 53, 730-735.
Riehle, A. (2005). Preparation for action: One of the key functions of the motor cortex. In A. Riehle \& E. Vaadia (Eds.), Motor cortex in voluntary movements: A distributed system for distributed functions (pp. 213-240). London: CRC Press.

Riehle, A., Grün, S., Diesmann, M., \& Aertsen, A. (1997). Spike synchronization and rate modulation differentially involved in motor cortical function. Science, 278, 1950-1953.

Roshan, L., Paradiso, G. O., \& Chen, R. (2003). Two phases of short-interval intracortical inhibition. Experimental Brain Research, 151, 330-337.

Rossini, P. M., Barker, A. T., Berardelli, A., Caramia, M. D., Caruso, G., Cracco, R. Q., et al. (1994). Non-invasive electrical and magnetic stimulation of the brain, spinal-cord and roots: Basic principles and procedures for routine clinical application. Electroencephalography and Clinical Neurophysiology, 91, 79-92.

Rossini, P. M., Zarola, F., Stalberg, E., \& Caramia, M. (1988). Pre-movement facilitation of motor-evoked potentials in man during transcranial stimulation of the central motor pathways. Brain Research, 458, 20-30.

Scheirs, J. G., \& Brunia, C. H. (1985). Achilles tendon reflexes and surface EMG activity during anticipation of a significant event and preparation for a voluntary movement. Journal of Motor Behavior, 17, 96-109.

Schoffelen, J. M., Oostenveld, R., \& Fries, P. (2005). Neuronal coherence as a mechanism of effective corticospinal interaction. Science, 308, 111-113.

Sohn, Y. H., Wiltz, K., \& Hallett, M. (2002). Effect of volitional inhibition on cortical inhibitory mechanisms. Journal of Neurophysiology, 88, 333-338.

Terao, Y., Ugawa, Y., Suzuki, M., Sakai, K., Hanajima, R., Gemba-Shimizu, K., et al. (1997). Shortening of simple reaction time by peripheral electrical and submotorthreshold magnetic cortical stimulation. Experimental Brain Research, 115, 541-545.

Thoenissen, D., Zilles, K., \& Toni, I. (2002). Differential involvement of parietal and precentral regions in movement preparation and motor intention. Journal of Neuroscience, 22, 9024-9034.

Touge, T., Taylor, J. L., \& Rothwell, J. C. (1998). Reduced excitability of the cortico-spinal system during the warning period of a reaction time task. Electroencephalography and Clinical Neurophysiology, 109, 489-495.

Trillenberg, P., Verleger, R., Wascher, E., Wauschkuhn, B., \& Wessel, K. (2000). CNV and temporal uncertainty with "ageing" and "non-ageing" S1-S2 intervals. Clinical Neurophysiology, 111, 1216-1226.

Yamanaka, K., Kimura, T., Miyazaki, M., Kawashima, N., Nozaki, D., Nakazawa, K., et al. (2002). Human cortical activities during Go/NoGo tasks with opposite motor control paradigms. Experimental Brain Research, 142, 301-307.

Ziemann, U., Rothwell, J. C., \& Ridding, M. C. (1996). Interaction between intracortical inhibition and facilitation in human motor cortex. Journal of Physiology, 497, 873-881.

Zijdewind, I., \& Kernell, D. (1994). Index finger position and force of the human first dorsal interosseus and its ulnar nerve antagonist. Journal of Applied Physiology, 77, 987-997. 